\title{
DEEP INELASTIC SCATTERING FROM QUANTUM FLUIDS
}

\author{
Victor K. WONG \\ Physics Laboratory, Department of Natural Sciences, \\ University of Michigan, Dearborn, MIchigan 48128, USA
}

Received 8 December 1976

Revised manuscript received 3 May 1977

\begin{abstract}
A simple and general argument is given for the deep inelastic behavior of the dynamic structure function of quantum fluids at zero temperature. The resulting high-energy tail is shown to give a reasonable fit to existing neutron data for liquid ${ }^{4} \mathrm{He}$.
\end{abstract}

Inelastic neutron scattering [e.g. 1] from quantum fluids at low energy transfers has been used extensively as a direct measure of the elementary (density) excitations characteristic of the quantum fluid. At higher energy transfer, more complicated features associated with multiexcitations appear and the simple picture of elementary excitations breaks down. At even higher energy transfer, it might be expected that the response of the quantum fluid would become independent of its elementary excitations, long-range order, particle statistics, etc., and would be understandable in terms of a universal picture. The purpose of this note is to propose such a simple and general picture of scattering at high energy transfer from quantum fluids at zero temperature.

The linear response of a quantum fluid to a density probe like neutron scattering is given by the dynamic structure function $S(k, \omega)$ defined as

$S(k, \omega)=\sum_{\nu}\left|\left(\rho_{k}^{\dagger}\right)_{\nu 0}\right|^{2} \delta\left(\omega-\omega_{\nu 0}\right)$.

The summation is over a complete set of normalized energy eigenstates $|\nu\rangle$ with total momentum $\boldsymbol{k}(\hbar=1)$; $|\nu\rangle$ is coupled to the ground state $|0\rangle$ by density fluctuations $\rho_{k}^{\dagger}$ with matrix elements $\left(\rho_{k}^{\dagger}\right)_{\nu 0}$; and $\omega_{\nu 0} \equiv$ $\omega_{\nu}-\omega_{0}$ is the excitation energy. Since the elementary excitations $|1\rangle$ exhaust the $f$ sum rule in the long wavelength limit, it is useful [2] to divide $|\nu\rangle$ into elementary excitations $|1\rangle$ and the remaining multiexcitations $|n\rangle$. In the region in $k, \omega$ space where ele-

\footnotetext{
* Supported in part by a Faculty Research Grant from the Horace H. Rackham School of Graduate Studies at the University of Michigan.
}

mentary excitations are not important, the background of $S(k, \omega)$, which arises from real multiexcitations, is given by scattering function

$$
X(k, \omega)=\sum_{n}\left|\left(\rho_{k}^{\dagger}\right)_{n 0}\right|^{2} \delta\left(\omega-\omega_{n 0}\right),
$$

where the sum is over the set of multiexcitations $|n\rangle$. The background region can be divided into the quasifree region $\omega \sim k^{2} / 2 m$ and the deep inelastic region $\omega \gg k^{2} / 2 m$. The deep inelastic region is of interest here.

The matrix elements $\left(\rho_{k}^{\dagger}\right)_{n 0}$ satisfy the equations

$$
\begin{aligned}
& \omega_{n 0}\left(\rho_{k}^{\dagger}\right)_{n 0}=k\left(j_{k}^{\dagger}\right)_{n 0}, \\
& \omega_{n 0}\left(j_{k}^{\dagger}\right)_{n 0}=\left[H, j_{k}^{\dagger}\right]_{n 0},
\end{aligned}
$$

where (3) is the continuity equation, (4) is the equation of motion for the longitudinal current $j_{k}^{\dagger}$, and $H$ is the many-particle hamiltonian for the quantum fluid. In the long wavelength limit $k \rightarrow 0,\left[H, j_{k}^{\dagger}\right]$ vanishes as a consequence of translational invariance. The leading $k$ dependence of $\left[H, j_{k}^{\dagger}\right]$ can then be obtained by a straightforward power series expansion:

$$
\left[H, j_{k}^{\dagger}\right]=m^{-1} \hat{\Phi} k+\mathrm{O}\left(k^{2}\right)
$$

where $m$ is the particle mass, and the $k$-independent operator $\hat{\Phi}$ is exhibited below. It follows from (2)-(5) that the leading $k$ dependence of $X(k, \omega)$ is given by

$$
\begin{aligned}
& X(k, \omega)=\frac{k^{4}}{m^{2} \omega^{4}} \sum_{n}\left|(\hat{\Phi})_{n 0}\right|^{2} \delta\left(\omega-\omega_{n 0}\right), \\
& \hat{\Phi}=\frac{1}{4} \sum_{i \neq j}\left(\hat{k} \cdot \hat{r}_{i j}\right)^{2} r_{i j} U^{\prime}\left(r_{i j}\right),
\end{aligned}
$$


where a term in $\hat{\Phi}$ that vanishes upon acting on $|0\rangle$ has been omitted, $r_{i j}$ is the position of the $i$ th particle relative to the $j$ th, $r_{i j}=\left|r_{i j}\right|, U$ is the interparticle potential, and $U^{\prime}(x) \equiv \mathrm{d} U(x) / \mathrm{d} x$.

Eqs. (6) and (7) are valid for small momentum transfer. To reduce (6) further, it is necessary to introduce a specific form for $\omega_{n 0}$. At high energy transfer, the multiexcitation energy is like that of free particles, e.g., $\omega_{20}=\epsilon_{k-q}+\epsilon_{q}$, where $\epsilon_{q} \equiv q^{2} / 2 m$ and $\boldsymbol{q}$ is the intermediate momentum. The contribution to $X(k, \omega)$ from pair excitations $|2\rangle$ can then be written ( $V$ is the volume)

$X_{2}(k, \omega)=\frac{k^{4}}{m^{2} \omega^{4}} V \int \frac{\mathrm{d}^{3} q}{(2 \pi)^{3}}\left|\hat{\Phi}_{20}\right|^{2} \delta\left(\omega-2 \epsilon_{q}\right)$,

which is valid in the deep inelastic region. To obtain the explicit form of $\hat{\Phi}_{20}$ and hence $X_{2}(k, \omega)$, we approximate the ground state $|0\rangle$ by $|\widetilde{0}\rangle$, the vacuum state of a model system of non-interacting excitations, and approximate $|2\rangle$ by $|\boldsymbol{k}-\boldsymbol{q}, \boldsymbol{q}\rangle \sim \rho_{k-q}^{\dagger} \rho_{q}^{\dagger}|\widetilde{0}\rangle$. Retaining only the terms with the same large intermediate momentum $\boldsymbol{q}$, we find

$$
\begin{aligned}
& X_{2}(k, \omega)=\frac{A}{320 \pi^{2}} \frac{N \rho k}{\omega^{2}}\left(\frac{k^{2}}{m \omega}\right)^{3 / 2} \varphi^{2}(\sqrt{m \omega}), \\
& \varphi(q) \equiv \int \mathrm{d}^{3} r(\hat{q} \cdot \hat{r})^{2} \mathrm{e}^{-\mathrm{i} q \cdot r}\left|r U^{\prime}(r)\right|,
\end{aligned}
$$

where $A$ is a dimensionless factor introduced to take into account the terms omitted in the approximation leading to (9). Since $q$ in $\varphi(q)$ is taken to be large in (9), the interparticle potential at small $r$ is important in (10) and the $r$-integral can be effectively cut-off at large $r$. If the potential is short-ranged at small $r$, e.g., $U(r) \sim \delta(r)$, then $\varphi(q)=$ constant and $X_{2}(k, \omega) \sim$ $k^{4} \omega^{-7 / 2}$. If the potential is long-ranged at small $r$, e.g., $U(r) \sim r^{-1}$, then $\varphi(q) \sim q^{-2}$ and $X_{2}(k, \omega) \sim$ $k^{4} \omega^{-11 / 2}$ as $\omega \rightarrow \infty$. Both of these results agree with previous work $[3,4]$. The present argument however is more general in that it is explicitly independent of the particular elementary excitations, long-range order, and particle statistics.

We shall now apply (9) and (10) to liquid ${ }^{4} \mathrm{He}$. The dominant interaction between ${ }^{4} \mathrm{He}$ atoms is the hard core, which produces a ground state $|0\rangle$ with, roughly speaking, excluded volumes. On the other hand, the model vacuum $|\widetilde{0}\rangle$ is one with uniform density. It is clear in this case that the approximation $|0\rangle \sim|\widetilde{0}\rangle$ is

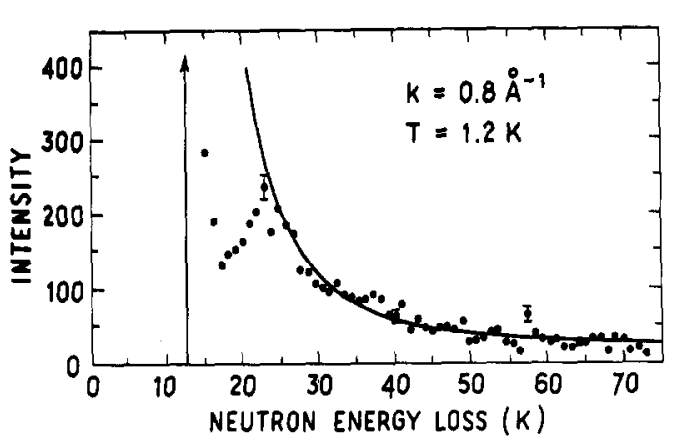

Fig. 1. The high energy tail in the intensity function of inelastic neutron scattering from liquid ${ }^{4} \mathrm{He}$ at a fixed momentum transfer $k$ and temperature $T$. The dots represent the neutron data from ref. [4], the vertical arrow denotes the elementary excitation peak, and the solid curve is the two-parameter fit (11).

not valid and that a straightforward substitution of a hard core potential into $(10)$ is misleading. These difficulties can be circumvented in the limit of small momentum transfer by the use of an effective potential $U_{\text {eff }} \sim \delta(r)$, where the proportionality constant is linear in the scattering length. It then follows from (9) and (10) that $X_{2}(k, \omega) \sim k^{4} \omega^{-7 / 2}$. The behavior however is not expected to persist at high energies when the hard core begins to soften. Calculation of $X_{3}(k, \omega)$ shows that the high- $\omega$ dependence of $X_{3}$ is slower than that for $X_{2}$. We therefore assume that for a finite interval in $\omega, X_{n>2}=X-X_{2}$ is more or less $\omega$-independent and forms a background for $X_{2}(k, \omega)$. In fig. 1 the intensity function $I(\omega)$ of neutron scattering [5] from liquid ${ }^{4} \mathrm{He}$ at fixed momentum transfer is plotted versus $\omega$. The solid curve is our twoparameter fit based on (9), the effective potential $U_{\text {eff }} \sim \delta(r)$, and the assumption that $X_{n>2}$ forms a constant background:

$I(\omega)=1.4 \times 10^{7} \omega^{-7 / 2}+25$,

where $\omega$ is measured in $\mathrm{K}$. The fit is seen to be fairly good in the range $25 \mathrm{~K}<\omega<70 \mathrm{~K}$, which is encouraging.

Without explicit reference to the particular elementary excitations, long-range order, and particle statistics of a quantum fluid, we have argued that deep inelastic scattering from a charged quantum fluid display a high- $\omega$ tail $\sim \omega^{-11 / 2}$; whereas deep inelastic scattering from a neutral quantum fluid, e.g., liquid 
${ }^{4} \mathrm{He}$, displays a tail $\sim \omega^{-7 / 2}$ over a finite high- $\omega$ range. Hence the relevant distinction ${ }^{\ddagger}$ is between charged and neutral quantum fluids, and within each class the deep inelastic scattering is expected to be universal, barring complications from multiexcitations higher than pair excitations.

₹ The relevant distinction in backflow is also between neutral and charged quantum fluids, as shown in ref. [2].

\section{References}

[1] A.D.B. Woods and R.A. Cowley, Rep. Prog. Phys. 36 (1973) 1135.

[2] V.K. Wong, J. Low Temp. Phys. 18 (1975) 65.

[3] V.K. Wong and H. Gould, Ann. Phys. (NY) 83 (1974) 252 ;

D.L. Bartley and V.K. Wong, Phys. Rev. B 12 (1975) 3775 .

[4] I. Family, Phys. Rev. Lett. 34 (1975) 1374.

[5] A.D.B. Woods, E.C. Svensson and P. Martel, Inelastic neutron scattering (Inter. Atomic Energy Agency, Vienna, 1973) p. 359. 